\title{
Comparative analysis of bilirubin in correlation to albumin between nephrotic syndrome patients and postoperative gastroparesis syndrome patients
}

M.H. Song, G.J. Zhu, L. Ma, G.L. Chen, D. Yang, J.H. Gong, Y.X. Xie, Y. Yan and M.C. Wang

Integrated Chinese and Western Medicine Treatment of Renal Disease Centre, Beidaihe Sanatorium of Beijing Military Area Command, Chinese PLA, Qinhuangdao, China

Corresponding author: L. Ma

E-mail: mhdoccn@126.com

Genet. Mol. Res. 13 (4): 9403-9411 (2014)

Received June 13, 2013

Accepted November 1, 2013

Published February 14, 2014

DOI http://dx.doi.org/10.4238/2014.February.14.13

\begin{abstract}
This study aimed to disclose the potential causality of low bilirubin in patients with nephrotic syndrome (NS). Correlation analysis was carried out on total bilirubin (TBIL) to serum albumin (ALB), urine protein $(\mathrm{Upr})$, and urinary microalbumin/creatinine $(\mathrm{Umalb} / \mathrm{cr})$ for three groups in a case-control study. $\mathrm{P}<0.001$ was observed for TBIL, ALB, Umalb/cr, and Upr between the NS and chronic nephritis $(\mathrm{CN})$ groups, and $\mathrm{P}$ values of $0.0001,1.000,0.0001$, and 0.0001 were observed for TBIL, ALB, Umalb/cr, and Upr, respectively, between the postoperative gastroparesis (PGS) and $\mathrm{CN}$ groups. The values of $r$ and $\mathrm{P}$ in correlation to TBIL were 0.549 and 0.000 for ALB, -0.405 and 0.000 for Umalb/cr, and -0.448 and 0.000 for Upr in the NS group; -0.007 and 0.959 for ALB, 0.213 and 0.091 for Umalb/cr, and -0.082 and 0.519 for Upr in the PGS group; and 0.509 and 0.000 for ALB, -0.431 and 0.000 for Umalb/cr, and -0.362 and 0.002 for $\mathrm{Upr}$ in the $\mathrm{CN}$ group. A probable
\end{abstract}


causality is implied between the low level of blood bilirubin and its loss in urine in NS patients. This conclusion may provide a theoretical basis for the feasibility of therapies against oxidative stress in NS patients.

Key words: Bilirubin; Nephrotic syndrome; Chronic nephritis; Urine protein; Albumin

\section{INTRODUCTION}

Bilirubin is mostly derived from degraded hemoglobin, and the rest is from the reticuloendothelial cells in the bone marrow, spleen, and liver. Bilirubin is generated under the effect of biliverdin reductase from dehydrobilirubin, a molecule from the oxidation of hemoglobin catalyzed by heme oxygenase in the presence of nicotinamide adenine dinucleotide phosphate and oxygen.

Schwertner et al. (1994) first recognized lower bilirubin levels in patients with coronary heart disease compared with healthy control individuals, which suggests that low bilirubin level is a newly found risk factor for coronary heart disease. Since this discovery by Schwertner et al., the biological effect of bilirubin has gained increasing attention.

Bilirubin contains an active hydrogen atom and elongated C-10 structure (i.e., a conjugated double-bond system). Moreover, bilirubin is a natural antioxidant and is one of the members of the endogenous antioxidant system similar to glutathione in the human body. Bilirubin can eliminate free radicals, inhibit lipid hyperoxidation, alleviate oxidative damage, and improve the antioxidation capability of patients. Bilirubin is a substance with biological antioxidation effect that protects the cerebral and cardiovascular systems under stress.

Bilirubin has a stronger antioxidation activity than vitamin E (Wu et al., 1996). Bilirubin may either directly or indirectly prevent low-density lipoprotein (LDL) from being oxidized by oxygen-derived free radicals produced by 2-2 nitrogen (2-propyl amidine) dihydrochloride at $37^{\circ} \mathrm{C}$, thereby effectively preventing the formation of oxidatively modified LDL (ox-LDL) (Mayer, 2000).

Urine protein (Upr) level higher than $3.5 \mathrm{~g} / \mathrm{dL}$ and plasma albumin concentration lower than $3 \mathrm{~g} / \mathrm{dL}$ are the necessary conditions for the diagnosis of nephrotic syndrome (NS), which is characterized by hyperlipidemia as a frequent clinical manifestation. Proteinuria is defined as the increase in protein amount in crude urine, which results from the damaged barrier of both molecules and loads provided for glomerular filtration membrane. When protein loss exceeds the reabsorption rate at the proximal tubule, high-grade proteinuria occurs. This condition is characterized as a discharge of protein and is complicated with the loss of a great deal of albumin in the urine, which would improve the compensatory synthesis of albumin by the liver. However, hypoproteinemia occurs when the enhanced synthesis of albumin cannot compensate its loss.

After being generated by the cells in the mononuclear phagocytic system and then released into circulation, bilirubin may be categorized into three groups according to its existing forms: free, combined, and covalent.

In the plasma, the existence and transportation of bilirubin are mainly in the form of a bilirubin-albumin complex. The combination of bilirubin and albumin in the plasma not only improves water solubility and transportation by plasma, but also limits the free penetration through membranes of various cells, thereby preventing toxic effects to cells and tissues. 
Studies have proven a high-affinity binding site and a low-affinity binding site on albumin for the covalent combination with bilirubin (Margarson and Soni, 1998).

Given the low plasma level bilirubin that is determined in normal clinical conditions as well as the combination of $25 \mathrm{mg}$ of bilirubin with every $100 \mathrm{~mL}$ plasma albumin, plasma albumin has a great tendency to combine with bilirubin and only a small amount of bilirubin remains unbound.

However, the combination form for most bilirubin with albumin is nonspecific, noncovalent, and reversible. Moreover, the combination rate is greater than $99 \%$ (Ostrow et al., 2003). Transfer of bilirubin to tissues and cells from plasma may be initiated by the decrease in albumin content, binding sites occupied by other substances, or lowered affinity of bilirubin to the sites.

Several organic anions, such as fatty acids and salicylic acid, may compete with bilirubin for albumin, thereby setting bilirubin free.

As a strong endogenous antioxidant in the human body, bilirubin is a main member of substances with antioxidant activity in serum and is capable of efficiently eliminating superoxide, peroxide, and free radicals. Bilirubin is important in diseases correlated to oxidative stress, including hypertension, coronary heart disease, and diabetes. Studies have indicated low levels of bilirubin in NS patients (Danesh et al., 2002; Usui et al., 2003). Therefore, we made a comparative study on bilirubin, serum albumin (ALB), and 24-h urinary protein quantity in three groups of patients with NS, chronic nephritis $(\mathrm{CN})$, and postoperative gastroparesis syndrome (PGS) $(\mathrm{ALB}<3.5 \mathrm{~g} / \mathrm{dL})$.

\section{MATERIAL AND METHODS}

\section{Subjects}

This case-control study was carried out under the guidance of the Ethics Committee of our hospital. Cases treated from January 2010 to February 2013, including 198 patients with NS, 70 with chronic nephritis, and 64 with PGS (ALB $<3.5 \mathrm{~g} / \mathrm{dL}$ ), who met the inclusion criteria were included in this research. General information about the subjects is listed in Table 1.

\begin{tabular}{|c|c|c|c|}
\hline & NS group & $\mathrm{CN}$ group & PGS group \\
\hline Case number & 198 & 70 & 64 \\
\hline Age (years) & $39.26 \pm 10.23$ & $41.14 \pm 13.84$ & $43.72 \pm 10.58$ \\
\hline Gender ratio & $102: 96$ & $36: 34$ & $35: 29$ \\
\hline Creatinine & $63.5 \pm 16.9$ & $178 \pm 44.6$ & $58.9 \pm 14.8$ \\
\hline Blood urea nitrogen & $6.01 \pm 2.48$ & $11.6 \pm 5.39$ & $5.26 \pm 2.59$ \\
\hline Triglycerides & $3.01 \pm 1.30$ & $2.48 \pm 1.75$ & $1.26 \pm 0.82$ \\
\hline Total cholesterol & $13.1 \pm 3.57$ & $5.82 \pm 1.58$ & $3.00 \pm 0.92$ \\
\hline Cystatin C (mg/L) & $0.86 \pm 0.23$ & $2.60 \pm 0.73$ & $0.92 \pm 00.19$ \\
\hline Glomerular filtration rate $\left(\mathrm{mL} \cdot \mathrm{min}^{-1} \cdot 1.73 \mathrm{~m}^{-2}\right)$ & $89 \pm 17$ & $47.8 \pm 13$ & $93 \pm 18$ \\
\hline
\end{tabular}

$\mathrm{NS}=$ nephrotic syndrome; $\mathrm{CN}=$ chronic nephritis; $\mathrm{PGS}=$ postoperative gastroparesis.

\section{Diagnostic criteria for PGS}

Given the lack of generally accepted criteria for the diagnosis of PGS worldwide, the generally adopted Bar-Natan et al. (1996) criteria were used as the basis in this research. 
PGS was established for a patient who manifested epigastric fullness, nausea, and vomiting in liquid diet or postconversion to a semi-liquid diet. Positive succussion splash should be found in the stomach percussion at day 7 or 8 after stomach surgery. The daily volume of gastric juice should be over $800 \mathrm{~mL}$ and should be mixed with bile that was drained for a prolonged period that lasted at least 8 to 10 days after reintubation was performed for gastrointestinal decompression. No postoperative medication that might interfere with the contraction of stomach smooth muscles should be taken. No obvious water/electrolytes disturbance or acid/base imbalance should be observed. The patient should have no underlying diseases that may cause gastroparesis, such as hypothyroidism and diabetes. No mechanical obstruction should be present in the outflow tract of stomach and ALB $<3.5 \mathrm{~g} / \mathrm{dL}$.

The diagnosis criteria for chronic nephritis include the main clinical manifestations of primary chronic nephritis, such as proteinuria, hypertension, edema, and hematuresis or cylindruria, as well as slow progression, prolonged course, varying severity, and with a duration of at least one year (Maciel et al., 2004).

The diagnosis criteria for NS include a urine protein level $>3.5 \mathrm{~g} / \mathrm{dL}$, plasma albumin content $<3.0 \mathrm{~g} / \mathrm{dL}$, edema, and hyperlipidemia.

\section{Exclusion criteria}

Patients with renal insufficiency, secondary glomerulopathy, diabetes, or diseases of the heart, cerebral vessels, liver, and hemopoietic system were excluded from the three groups.

\section{Laboratory analysis}

An automated biochemical analyzer (AU640, Olympus Optical Co., Tokyo, Japan) was used to determine total cholesterol, triglycerides, total bilirubin (TBIL), direct bilirubin, and ALB. All assay kits were purchased from Randox Company (Crumlin, UK). The procedures were strictly performed in accordance with the instructions. A 24-h urine protein quantitative assay was carried out using the biuret method. The sample was collected prior to renal puncture. First, a qualitative assay was performed prior to spiking, and a five-time dilution was made for a sample yielding results stronger than +++ .

\section{Statistical analysis}

The Kruskal-Wallis rank sum test was performed for inter-group comparison on each parameter, Bonferroni's method for two-by-two comparisons, and the Pearson test for correlation of TBIL with Upr and ALB. Statistical tests were performed using SPSS 11.0, with $\mathrm{P}<$ 0.05 regarded as significant.

\section{RESULTS}

\section{Characteristics at baseline}

No significant difference was observed in the population characteristics between NS and PGS, and the characteristics seemed comparable with each other (Table 1). 


\section{Levels of BTIL, Upr, and LB}

The values of $\mathrm{H}$ and $\mathrm{P}$ in the $\mathrm{CN}$ group compared with the NS and PGS groups were 81.92 and 0.000 for TBIL, 120.49 and 0.000 for ALB, 148.12 and 0.000 for urinary microalbumin/creatinine (Umalb/cr), and 160.38 and 0.000 for Upr. The values of $Z$ and $P$ were 4.77 and 0.0001 for TBIL, 11.03 and 0.0001 for ALB, 2.88 and 0.013 for Umalb/cr, and 3.79 and 0.0005 for Upr in comparison between NS and CN, whereas the values were 4.61 and 0.0001 for TBIL, 0.01 and 1.000 for ALB, 11.22 and 0.0001 for Umalb/cr, 11.57 and 0.0001 for Upr in comparison between PGS and CN (Table 2).

\begin{tabular}{|c|c|c|c|c|}
\hline Group & ALB & TBIL & $\mathrm{Upr} / 24 \mathrm{~h}$ & Umalb/cr \\
\hline NS & $22.79 \pm 7.01$ & $6.54 \pm 3.52$ & $4.53 \pm 2.79$ & $438.83 \pm 305.22$ \\
\hline $\mathrm{CN}$ & $32.08 \pm 5.88^{*}$ & $9.14 \pm 5.19^{*+}$ & $3.88 \pm 4.61^{*+}$ & $355.78 \pm 300.47^{*+}$ \\
\hline PGS & $31.46 \pm 3.25^{*}$ & $15.24 \pm 7.87^{*}$ & $0.05 \pm 0.02 *$ & $1.01 \pm 0.59^{*}$ \\
\hline
\end{tabular}

\section{Analysis on correlation}

The values of $\mathrm{r}$ and $\mathrm{P}$ in correlation to TBIL were 0.549 and 0.000 for ALB, -0.405 and 0.000 for Umalb/cr, and -0.448 and 0.000 for Upr in the NS group; -0.007 and 0.959 for ALB, 0.213 and 0.091 for Umalb/cr, and -0.082 and 0.519 for Upr in the PGS group; and 0.509 and 0.000 for ALB, -0.431 and 0.000 for Umalb/cr, and -0.362 and 0.002 for Upr in the CN group (Figure 1).

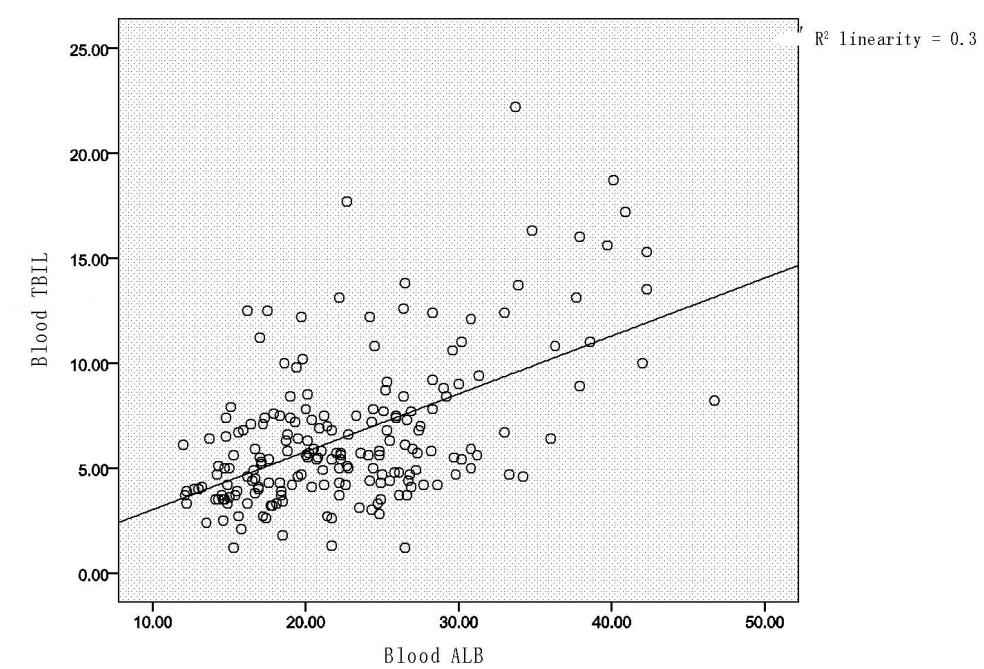

Figure 1. Scatter diagram of the correlation between blood total bilirubin (TBIL) and albumin (ALB) in NS patients.

\section{DISCUSSION}

This research aimed to provide a theoretical basis for therapies against oxidative stress 
in NS patients and to discuss further the main reason for their low level of blood bilirubin through a comparative analysis of its level between NS, chronic nephritis, and gastroparesis groups. The control subjects were patients with PGS with hypoproteinemia, and correlation analysis was performed on the blood bilirubin and albumin of these parameters in NS patients.

PGS is one of the sequelae of surgeries on the abdomen (Parkman, 2009). Sympathetic activity-boosted postabdominal surgery may direct the stomach wall to release norepinephrine or inhibitory substances, either of which may act directly upon the $\alpha$ or $\beta$ receptor on gastrointestinal smooth muscles to block the release of acetylcholine by parasympathetic neurons, thereby inhibiting the myoelectric activity of the stomach and prolonging gastric emptying (Livingston and Passaro, 1990).

The excision of the antrum and pylorus, the most active parts in the stomach, makes coordinated movement involving antrum, pylorus, and duodenum impossible after subtotal gastrectomy and weakens the functions of the gastric remnant $(\mathrm{Lu}, 1999)$. This condition leads to malnutrition and hypoproteinemia.

Our results showed that bilirubin was not correlated with blood albumin. In the gastroparesis group, either the 24-h urine protein quantity or the analysis of trace urine protein showed a negative finding, and no correlation was observed in blood bilirubin with either 24-h urine protein qualitative assay or trace urine protein. In the NS and chronic nephritis groups, however, bilirubin was positively correlated with blood ALB and negatively correlated with Upr and trace urine protein. This result seemed consistent with those of previous reports (Fukui et al., 2008), which suggests that the low level of bilirubin may be correlated with hypoproteinemia or loss of bilirubin through urine because of proteinuria.

Given the absence of significant differences in blood albumin between the chronic nephritis group and gastroparesis group, a comparative analysis was carried out between these two groups to determine if blood bilirubin was correlated with blood albumin. In the NS and the chronic nephritis groups, however, blood bilirubin was correlated to either urine protein quantity or trace urine protein, but not to blood albumin. This result suggests the main causality between the loss of bilirubin in the urine and its low level in blood in the NS group.

Metabolism disorder of blood lipids is one of the basic manifestations of NS. An incidence beyond $70 \%$ of hyperlipidemia has been reported in NS patients by Appel (1991) and Skrzep-Poloczek et al. (2001). Increased cell numbers in the glomerulus at week 4 has been proven by a previous research in hypercholesterolemic rats, in contrast to the obviously decreased number at week 8 with increasing urine protein. This result suggests the importance of lipids in causing glomerulus injuries (Hattori et al., 1998). The significantly greater infiltration by inflammatory cells in kidney tubules and mesenchyme than in tubules, as shown by Eddy (1998) in mice fed with high-cholesterol forage for 4 weeks, implies the presence of injury in the tubular endoplasmic reticulum. The fibrosis in mesenchyme observed at week 8 in the same study suggests the alteration in both tubules and the correlation of its mesenchyme with kidney injuries caused by lipids.

Since the discovery of the antioxidant features of bilirubin, functions against oxidative free radicals have been proven in all members of the bilirubin family (Mayer, 2000). Olinescu et al. (1999) considered that the activating capability of hemoglobin may dramatically decrease against oxidative stress in aged patients than in young ones, and this capability is negatively correlated with bilirubin (Skrzep-Poloczek et al., 2001). In addition, oxygen-free radicals that are systematically produced are also considered to aggravate the injuries to glo- 
merular capillary walls (Kinra et al., 2000).

Bilirubin is a potential, endogenous antioxidant because its activity against oxidation is superior to those of vitamins $\mathrm{C}$ and $\mathrm{E}$ and carotene, as suggested by previous studies (Wu et al., 1996; Ihara et al., 2004). Bilirubin may improve cellular resistance against oxygen attack and block the production of ox-LDL, which has an important function in the progression of atherosclerotic plaque. By altering the morphology and structure of endothelial cells, ox-LDL destroys the structure of the vascular endothelium, increases the expression of cell adhesion molecules in large quantities, and allows monocytes to enter the subintimal layer, where they differentiate into macrophages. These macrophages take up lipids by using their scavenger receptors on their surface to form foam cells (Ujhelyi et al., 2006).

Bilirubin is a biological antioxidant and cellular protectant (Mayer, 2000). The asymmetry of albumin on bilirubin drives the hydrogens on the $\mathrm{C} 10$ of bilirubin to transform into active hydrogen atoms, which are easily combined with free radicals, such as superoxide anions, to eliminate oxygen-free radicals. The strong antioxidant effect of bilirubin, which can be realized by the contact expansion of biliverdin reductase, contributes to $30 \%$ of all antioxidant capability in the human body and has a function in protecting the cardiovascular system from oxidative stress.

All bilirubin in serum may efficiently eliminate oxygen-free radicals and prevent lipid oxidation because of its strong antioxidant capability. This characteristic is an important pathological basis for the formation of atherosclerotic plaque (Djoussé et al., 2001). Bilirubin may enhance the activity of heme oxygenase, stimulate vascular endothelial cells to excrete a proper amount of nitrogen monoxide, dilate blood vessels, block ox-LDL-induced chemotaxis and monocyte deposits, and arrest the growth of smooth muscle cells in the blood vessels between G1- and S-phases (Basuroy et al., 2006). A research in a Korean population revealed a negative correlation between the concentration of plasma bilirubin and the incidence of hypertension (Juncos et al., 2008).

A research has also shown that hemoglobin applied to a hypertension model induced by deoxycorticosterone may lower blood pressure by upregulating HO-1, decreasing extracellular matrix protein (Jadhav et al., 2008), alleviating pathological injury to the myocardium and inhibiting ventricular remodeling. These authors also carried out an observation that lasted 3 months on the therapeutic effect of hemoglobin to reveal its continuous protection on myocardium, which is anticipated to be helpful in clinical treatment (Ndisang and Jadhav, 2009). After the injection of a free radical scavenger, a decrease in urine protein and an amelioration of glomerulus damage caused by oxygen-free radicals were observed by Someya et al. (2005) in animals with artificially induced minimal lesion nephrotic syndrome.

Bilirubin has important biological effects on lipid metabolism, elimination of free radicals, alleviation of proteinuria, and protection of glomerulus cells. If the level of bilirubin, the reducer, is elevated, the remission of NS may be improved, which provides a new method and a new idea for the treatment of NS. Therefore, the elucidated causality of low level of bilirubin in NS patients provides a theoretical basis for the feasibility of either a therapy involving a blockade of the lowering of bilirubin or another with an improvement of its level to eliminate free radicals in NS patients, inhibit the formation of ox-LDL, and protect their glomerular cells.

The potential reason for low bilirubin level in NS patients was explored in this research through a comparative analysis of bilirubin in correlation to blood albumin and urine protein between the gastroparesis and chronic nephritis groups. However, given the impossibility of precisely determining the amount of urinary bilirubin because of the presence of multiple confounding factors in determining its level, further research is needed to validate this 
probable causality between low blood bilirubin and its loss in the urine in NS patients based on a perfected procedure for determination, and approaches with which bilirubin concentration can be increased to a proper level for NS patients should be explored.

In short, our findings suggest that the low level of bilirubin in NS patients positively correlates with blood albumin and negatively correlates with either urine protein or urine trace albumin. In addition, a comparative analysis between patients with gastroparesis syndrome and those with chronic nephritis implied a probable causality between low level of bilirubin in blood and its loss through the urine in NS patients, which provides a theoretical basis for the feasibility of a therapy against oxidative stress in NS patients.

\section{REFERENCES}

Appel G (1991). Lipid abnormalities in renal disease. Kidney Int. 39: 169-183.

Bar-Natan M, Larson GM, Stephens G and Massey T (1996). Delayed gastric emptying after gastric surgery. Am. J. Surg. 172: 24-28.

Basuroy S, Bhattacharya S, Tcheranova D, Qu Y, et al. (2006). HO-2 provides endogenous protection against oxidative stress and apoptosis caused by TNF-alpha in cerebral vascular endothelial cells. Am. J. Physiol. Cell Physiol. 291: C897-C908.

Danesh FR, Sadeghi MM, Amro N, Philips C, et al. (2002). 3-Hydroxy-3-methylglutaryl CoA reductase inhibitors prevent high glucose-induced proliferation of mesangial cells via modulation of Rho GTPase/ p21 signaling pathway: Implications for diabetic nephropathy. Proc. Natl. Acad. Sci. U. S. A. 99: 8301-8305.

Djoussé L, Levy D, Cupples LA, Evans JC, et al. (2001). Total serum bilirubin and risk of cardiovascular disease in the Framingham offspring study. Am. J. Cardiol. 87: 1196-1200.

Eddy AA (1998). Interstitial fibrosis in hypercholesterolemic rats: role of oxidation, matrix synthesis, and proteolytic cascades. Kidney Int. 53: 1182-1189.

Fukui M, Tanaka M, Shiraishi E, Harusato I, et al. (2008). Relationship between serum bilirubin and albuminuria in patients with type 2 diabetes. Kidney Int. 74: 1197-1201.

Hattori T, Shindo S and Kawamura H (1998). Apoptosis and expression of Bax protein and Fas antigen in glomeruli of a remnant-kidney model. Nephron 79: 186-191.

Ihara H, Hashizume N, Hasegawa T and Yoshida M (2004). Antioxidant capacities of ascorbic acid, uric acid, alphatocopherol, and bilirubin can be measured in the presence of another antioxidant, serum albumin. J. Clin. Lab. Anal. 18: 45-49.

Jadhav A, Torlakovic E and Ndisang JF (2008). Interaction among heme oxygenase, nuclear factor-kappaB, and transcription activating factors in cardiac hypertrophy in hypertension. Hypertension 52: 910-917.

Juncos JP, Tracz MJ, Croatt AJ, Grande JP, et al. (2008). Genetic deficiency of heme oxygenase-1 impairs functionality and form of an arteriovenous fistula in the mouse. Kidney Int. 74: 47-51.

Kinra S, Rath B and Kabi BC (2000). Indirect quantification of lipid peroxidation in steroid responsive nephrotic syndrome. Arch. Dis. Child. 82: 76-78.

Livingston EH and Passaro EP (1990). Postoperative ileus. Dig. Dis. Sci. 35: 121-132.

Lu PW (1999). Diagnosis and treatment of gastroparesis after greater part gastrectomy. Chin. J. Bases Clin. Gen. Surg. 6: $173-174$.

Maciel GA, Baracat EC, Benda JA, Markham SM, et al. (2004). Stockpiling of transitional and classic primary follicles in ovaries of women with polycystic ovary syndrome. J. Clin. Endocrinol. Metab. 89: 5321-5327.

Margarson MP and Soni N (1998). Serum albumin: touchstone or totem? Anaesthesia 53: 789-803.

Mayer M (2000). Association of serum bilirubin concentration with risk of coronary artery disease. Clin. Chem. 46: 17231727.

Ndisang JF and Jadhav A (2009). Upregulating the heme oxygenase system suppresses left ventricular hypertrophy in adult spontaneously hypertensive rats for 3 months. J. Card. Fail. 15: 616-628.

Olinescu RM, Kummerow FA, Greabu M, Crocnan DO, et al. (1999). The levels of bilirubin may be related to an inflammatory condition in patients with coronary heart disease. Rom. J. Intern. Med. 37: 239-249.

Ostrow JD, Pascolo L, Shapiro SM and Tiribelli C (2003). New concepts in bilirubin encephalopathy. Eur. J. Clin. Invest. 33: 988-997.

Parkman HP (2009). Assessment of gastric emptying and small-bowel motility: scintigraphy, breath tests, manometry, and 
SmartPill. Gastrointest. Endosc. Clin. N. Am. 19: 49-55.

Schwertner HA, Jackson WG and Tolan G (1994). Association of low serum concentration of bilirubin with increased risk of coronary artery disease. Clin. Chem. 40: 18-23.

Skrzep-Poloczek B, Tomasik A, Tarnawski R, Hyla-Klekot L, et al. (2001). Nephrotic origin hyperlipidemia, relative reduction of vitamin $\mathrm{E}$ level and consequent oxidative stress may promote atherosclerosis. Nephron 89: 68-72.

Someya T, Kaneko K, Yamada T and Yamashiro Y (2005). Effect of a novel free radical scavenger, edaravone, on puromycin aminonucleoside induced nephrosis in rats. Pediatr. Nephrol. 20: 1430-1434.

Ujhelyi L, Balla G, Jeney V, Varga Z, et al. (2006). Hemodialysis reduces inhibitory effect of plasma ultrafiltrate on LDL oxidation and subsequent endothelial reactions. Kidney Int. 69: 144-151.

Usui H, Shikata K, Matsuda M, Okada S, et al. (2003). HMG-CoA reductase inhibitor ameliorates diabetic nephropathy by its pleiotropic effects in rats. Nephrol. Dial. Transplant. 18: 265-272.

Wu TW, Fung KP, Wu J, Yang CC, et al. (1996). Antioxidation of human low density lipoprotein by unconjugated and conjugated bilirubins. Biochem. Pharmacol. 51: 859-862. 